\title{
Guilt aversion and reciprocity in the performance enhancing drug game
}

\author{
Edward Cartwright ${ }^{*}$
}

June 29, 2018

\begin{abstract}
JEL Codes: Z20; C72;
Keywords: Doping; performance enhancement; game theory; guilt aversion; reciprocity; inequality aversion
\end{abstract}

\begin{abstract}
We revisit the performance enhancing drug game by applying models of guilt aversion and reciprocity. Both models fit within the framework of psychological game theory in that they allow payoffs to depend on beliefs. We explore the extent to which social norms can help reduce or eliminate doping in sport. With reciprocity we see that first-order beliefs on the prevalence of doping are key and a norm of clean sport would require a coordinated shift in such beliefs. With guilt aversion, by contrast, second-order beliefs are key and individuals may have an incentive to race clean even if they expect competitors will dope. Our results point to the importance for sports bodies and coaches to manage the beliefs of athletes.
\end{abstract}

*Department of Strategic Management and Marketing, De Montfort University, Leicester, LE1 9BH, UK. edward.cartwright@dmu.ac.uk

${ }^{\dagger}$ I would like to thank two anonymous reviewers and the editor of the Journal for their very detailed and constructive comments on earlier versions of the paper. 


\section{Introduction}

Many have argued that norms of fair play are crucial in efforts to eliminate or reduce doping in sport (e.g. Bird and Wagner 1997; Eber 2011). A particularly important contribution to the literature is provided by Eber (2008) who incorporates fair play norms into the performance-enhancing drug game of Haugen (2004) (which is itself closely related to games considered by Breivik 1992 and Berentsen 2002). Eber (2008) shows that a norm against obtaining an unfair advantage can fundamentally change incentives within the game and convert it from a prisoners dilemma game (with a unique Nash equilibrium of doping) to a coordination game (with a Nash equilibrium of no doping). Consistent with this approach, abundant survey evidence shows, as I will shortly review, that doping intentions are strongly correlated to an athlete's moral and normative views concerning doping (e.g Kavussanu 2008; Morente-Sánchez and Zabala 2013; Ntoumanis et al. 2014).

Taking Eber (2008) as my starting paint, the current paper is motivated by two basic observations. First, the literature on social norms has highlighted many different channels through which norms may work (Bicchieri 2018). For instance, a norm may be driven by fear that transgressions will be punished, or it could be driven by avoidance of guilt. Moreover, an extensive experimental literature has shown that beliefs, intentions, and beliefs about intentions are an important part of the mix alongside actions (e.g. Falk, Fehr and Fischbacher 2008). It is important, therefore, to consider a range of different models of social norms and social preferences to see if the results of Eber (2008) are sensitive to specific modeling assumptions. In the current paper we compare and contrast three seminal models of social preferences. We will see that this gives new insight and a more complete picture of how norms can influence doping.

That brings us to the second observation motivating this paper. The results of Eber (2008) suggest some significant hurdles have to be overcome if a no doping equilibrium is going to emerge. Most importantly, all athletes have to feel negatively about gaining an unfair advantage. If one athlete is selfish than any no doping equilibrium unravels. This means it is vital to explore whether norms of fair play, and the strong moral and normative views we know many athletes hold about doping, can realistically reduce doping in sport. The analysis to follow shows that the specific mechanism driving the social norm is crucial. In particular, if guilt is the main driving force then there are less hurdles to overcome in trying to reduce doping. This, as I shall discuss, has practical policy implications.

The preceding discussion briefly picked up the role of beliefs and intentions. This will be a key theme in the paper and so to introduce the basic issues consider the norm 'do not dope if others do not dope'. Realistically, an athlete does not know for sure the actions of others and so he simply does not know whether they will dope or not. He must, therefore, base his actions on beliefs about the actions of others. For instance, the norm could be written 'I will not dope if I believe others are not doping'. Or it might be that 'I will not dope if I believe that others expect me to not dope'. Moreover, an athlete may care about the intentions behind doping. For example, that a competitor dopes to gain an unfair advantage may seem worse than a competitor who dopes because they believed others would try to gain an advantage. This means we need to capture beliefs about intentions (Rabin 1993). In exploring the role of beliefs we also, crucially, need to recognize that beliefs may 
be incorrect. For instance, an athlete may overestimate the prevalence of doping (Petroczi et al. 2008). Ideally, therefore, we need to directly capture beliefs and recognize that the beliefs people act upon may be wrong.

Psychological game theory allows for payoffs to depend on both actions and beliefs (Geanakopolos, Pearce and Stacchetti 1989; Battigalli and Dufwenberg 2009) and so provides a general framework with which to model norms of fairness and reciprocity (e.g. Rabin 1993; Dufwenberg and Kirchsteiger 2004; Falk and Fischbacher 2006). In the current paper I apply two seminal models from psychological game theory, one based on guilt aversion (Charness and Dufwenberg 2006; Battigalli and Dufwenberg 2007, 2009; see also Bacharach et al. 2007) and another based on reciprocity (Rabin 1993; Dufwenberg and Kirchsteiger 2004). These models not only allows us to capture beliefs but also to explore the implications of incorrect beliefs. For instance, in the case of reciprocity it would seem possible, a-priori, that an athlete may feel less negatively about a competitor who dopes if he believes that this competitor expected others to dope. In this case the athlete expects his competitor to have wrong beliefs (that others will dope) and would judge 'fairness' relative to those wrong beliefs. We can only pick up such possibilities by explicitly taking beliefs into account, as in psychological game theory.

Consistent with the findings of Eber (2008) I find that both guilt aversion and reciprocity can result in an equilibrium with no doping. As already previewed, however, specific predictions depend crucially on whether guilt aversion or reciprocity is the driving factor. Guilt aversion focuses on second-order beliefs in saying that an athlete will race clean if he believes that others expect him to do so. We shall see that this can provide a strong incentive to race clean. Indeed, guilt aversion can mean it is optimal for an athlete to race clean irrespective of what others do. Reciprocity, by contrast, focuses on first-order beliefs in saying that an athlete will race clean if he believes others will do so. We shall see that this results in a weaker incentive to race clean and in this sense provides results closer to those of Eber (2008).

The implications of these findings will be discussed more as we proceed. It is worth noting, however, that both guilt aversion and reciprocity are likely to be important motives driving behavior (Dufwenberg, Gächter and Hennig-Schmidt 2011). A main contribution of the current paper is, therefore, to highlight the importance of beliefs for doping in sport. To illustrate the potential implications let me begin by highlighting that current estimates on the prevalence of doping vary enormously (Dilger, Frick and Tolsdorf 2007). Indeed, it would not be pushing things too much to say that all we know for certain is that the proportion of athletes who dope is somewhere between 0 and 100 percent. Clearly, such uncertainty gives considerable leeway for athletes to form their own beliefs.

In order to minimize doping we need athletes to have relatively optimistic firstorder beliefs. In an environment, however, where the media and authorities focus on doping scandals it seems almost inevitable that athletes will form relatively pessimistic first-order beliefs. Anshel (1991), for instance, found that the media's reporting of drug use by 'established sports stars' influenced general use of performance enhancement. It may, therefore, be important to 'manage' the beliefs of athletes. In a variety of contexts, including attitudes to body-image and alcohol consumption, it has been shown that 'correcting' erroneous beliefs can change behavior (Perkins 2002; Bergstrom and Neighbors 2006). This would suggest a role for 
athletes, coaches and the sporting authorities to create a more balanced impression on doping (Englar-Carlson et al. 2016). If a young athlete is surrounded by stories of doping then it would not be a surprise for that athlete to form pessimistic beliefs and consequently go down a doping route.

Care is also needed in terms of second-order beliefs. For an interesting example consider the current scandal engulfing Russian sport (McClaren 2016). The basic impression being created is one in which all Russian athletes dope. A young Russian athlete may clearly interpret this as a signal that others will think she is doping (whatever she does). In other words she will form pessimistic second-order beliefs. This, in turn, provides little incentive for her to race clean. Again, therefore, it would seem important that those involved in sport create an environment that emphasizes clean sport rather than overly dwelling on evidence of doping.

The paper proceeds as follows. In Section 2 I briefly review the literature connecting norms and moral beliefs to doping. In Section 3 the performance-enhancing drug game is introduced. For completeness, Section 4 analyzes a model of inequality aversion (Fehr and Schmidt 1999) that is in modeling terms identical to that of Eber (2008). Section 5 analyzes a model of reciprocity and Section 6 a model of guilt aversion. Section 7 concludes.

\section{Doping, norms and beliefs}

There is an extensive literature looking at the factors which influence an athlete's decision to dope. Given that there are two recent, excellent reviews of this literature, namely Morente-Sánchez and Zabala (2013) and Ntoumanis et al. (2014), it is not my intension here to survey that literature in any great depth. Instead I want to pick out results from the literature that seem particularly pertinent to the role that beliefs and norms can play in doping.

Let me begin with a quote from Strelan and Boeckmann (2006: p. 2912) that nicely sets the seen: '[T]he results of more than 50 perceptual-deterrence studies conducted over a range of unlawful behaviors from the petty (e.g. underage drinking, marijuana use) to the serious (e.g. rape, domestic abuse, drunk driving, tax fraud) have consistently shown that legal-sanction threats have little or no impact on decisions to engage in criminal acts. Rather, individuals are most likely to be deterred from illegal activity by their moral beliefs and their fear of social disapproval.' This passage refers to studies that did not directly look at doping in sport. The consistent picture that emerges, though, from the study of Strelan and Boeckmann (2006) and others is that doping in sport is no different. Moral beliefs and norms (together with health concerns) consistently show up as the major deterrents against doping.

This overall finding points to the critical role that norms and beliefs can play (and arguably must play) in combating doping. And note that much of the literature directly studies elite athletes and so the evidence is robust to this, non-random, subset of the population (Morente-Sanchez and Zabala 2013). Many different notions of moral beliefs and norms are, though, considered in the literature (see, for example, Kavussanu 2008 and Ntoumanis et al. 2014). So, in motivating the analysis of the current paper, I want to decompose the overall finding that norms matter and distinguish the different channels through which this can manifest itself. And I particularly want to distinguish the role of first and second-order beliefs. I will start 
by looking at second-order beliefs because this is where most evidence exists.

Recall that second-order beliefs capture an athlete's expectation about what others expect her to do. In short, does she think others expect her to dope? Such beliefs, as we shall see in Section 5, are critical in models of guilt aversion and moral sanctioning. A wide range of studies have found that guilt (sometimes subsumed under moral beliefs), together with the likely response of friends, colleagues and the public, are strong indicators of doping perception and intentions (e.g. Goulet et al. 2010; Overbye Knudsen and Pfister 2013; Jalleh, Donovan and Jonling 2014). It is important to highlight, however, that there are many channels through which this influence can materialize. For instance, Strelan and Boeckmann (2006) find that guilt has a strong effect, while the reaction of an important other, teammate or the public has a milder effect. Other studies, by contrast, have found a strong effect from the likely reaction of those close to the athlete (Overbye et al. 2013) or of the general public (Huybers and Mazanov 2012).

First-order beliefs capture an athlete's expectation about the likely behavior of other athletes. In short, are others going to dope? Such beliefs, as we shall see in Section 6, naturally feed into models of reciprocity and fairness. There are surprisingly few studies that directly elicit first-order beliefs and so the evidence here is necessarily weaker. Even so, there is consistent evidence that attitudes to doping are influenced by the likely prevalence of doping amongst competitors (e.g. Petróczi et al. 2008; Bloodworth and McNamee 2010; Kirby, Moran and Guerin 2011; Barkoukis, Lazurus and Tsorbatzoudis 2014). Specifically, there is a positive correlation between beliefs on the doping of others and own intention to dope.

The preceding discussion suggests that both first and second-order beliefs are likely to play an important role in an athlete's decision to dope. But, can we say which has the stronger effect? The study of Barkoukis et al. (2014) is particularly relevant in this regard as it allows a direct comparison of first-order beliefs (which they refer to as descriptive norms) and second-order beliefs (subjective norms). ${ }^{1}$ While both first and second order beliefs are found to be predictors of doping susceptibility, second order beliefs are the stronger predictor. Similarly, Overbye et al. (2013) found that the opinion, doping would be 'unfair to competitors who do not dope' was important but less so than guilt and the opinions of others. Also, 'competitors have started to use doping' was less important than things like potential medical supervision and benefits. Both of these studies suggest that first-order beliefs may be less of an influence than second-order beliefs. This could be crucial, as I discuss more in the conclusion.

Before we proceed to the formal analysis let me make the fairly obvious caveat that the analysis will not be able to capture all the subtle ways in which norms and beliefs may impact on the doping decision. Instead I shall focus on the rather blunt instrument of first and second-order beliefs in a two player game. Moreover, I shall not be able to take account of all the many different ways in which social norms can be modeled. In analyzing and comparing three seminal and very widely used models of social norms we do, though, gain critical new insight on the role that norms and

\footnotetext{
${ }^{1}$ To measure descriptive norms subjects are asked questions of the form 'How many athletes at your competitive level do you believe engage in doping to enhance their performance'. This is a direct elicitation of first-order beliefs. To measure subjective norms subjects are asked questions of the form 'Most people I know would approve of me using prohibited substances to enhance my performance during this season'. This is a direct elicitation of second-order beliefs.
} 
beliefs can play in doping decisions.

\section{The Performance-Enhancing Drug Game}

Consider the following doping game studied by Haugen (2004) and Eber (2008). ${ }^{2}$ There are two, risk neutral, athletes $(i=1,2)$ involved in a competition. They simultaneously and independently of each other must decide whether to dope $(D)$ or not dope $(N D){ }^{3}$ The prize for winning the competition is $w>0$ and the cost of doping is $h \geq 0$. In interpretation $h$ may include health costs or potential sanctions for being caught doping. If neither athlete dopes, or both athletes dope, then each has probability $1 / 2$ of winning the prize. If an athlete dopes and his competitor does not then his probability of winning the prize increases from $1 / 2$ to $p>1 / 2$. The corresponding payoffs to each of the four possible outcomes are detailed in Table 1.

Athlete 2

$\mathrm{D} \quad \mathrm{ND}$

Athlete $1 \quad \mathrm{D} \quad w / 2-h, w / 2-h \quad p w-h,(1-p) w$

$\mathrm{ND} \quad(1-p) w, p w-h \quad w / 2, w / 2$

Table 1: The performance-enhancing drug game payoff matrix

An action profile $a=\left(a_{1}, a_{2}\right)$ details the actions of each athlete where $a_{i} \in[0,1]$ is the probability that athlete $i=1,2$ dopes. In order to apply psychological game theory we need to take account of first-order and second-order beliefs. Let $b_{i j} \in[0,1]$ denote athlete $i$ 's belief about $a_{j}$. In other words, $b_{i j}$ is athlete $i$ 's belief about the probability his competitor will dope. Also, let $c_{i j i} \in[0,1]$ denote athlete $i$ 's belief about $b_{j i}$. In other words $c_{i j i}$ is athlete $i$ 's belief about athlete $j$ 's first-order belief. For instance, if $b_{i j}=1$ and $c_{i j i}=0$ then athlete $i$ believes athlete $j$ will dope and he believes that athlete $j$ believes he will not dope.

Let $u_{i}(a)$ denote the von-Neumann Morgenstern expected payoff of athlete $i=$ 1,2 given action profile $a$. Action profile $a^{*}=\left(a_{1}^{*}, a_{2}^{*}\right)$ is a Nash equilibrium if $u_{1}\left(a_{1}^{*}, a_{2}^{*}\right) \geq u_{1}\left(a_{1}, a_{2}^{*}\right)$ for all $a_{1} \in[0,1]$ and $u_{2}\left(a_{1}^{*}, a_{2}^{*}\right) \geq u_{2}\left(a_{1}^{*}, a_{2}\right)$ for all $a_{2} \in[0,1]$. In other words, at a Nash equilibrium, neither athlete can gain from unilateral deviation. It is trivial to see that if $(p-1 / 2) w>h$ then both athletes have a dominant strategy to dope. This means that action profile $(1,1)$ is the unique Nash equilibrium. It is also trivial that action profile $(0,0)$ is Pareto efficient and maximizes total payoff. The game has, therefore, the structure of a prisoners' dilemma (Haugen 2004). In the following we restrict attention to the case where $(p-1 / 2) w>h$.

Let me remark that the performance-enhancing drug game assumes symmetry in ability, gains from doping and costs of doping. This simplifying assumption can be justified on the grounds that we are focusing on two closely matched athletes who, because they are so closely matched, have a strong incentive to dope. For instance,

\footnotetext{
${ }^{2}$ For analysis of closely related games see Breivik (1992) and Berentsen (2002). For analysis of a many player version of the game see Haugen, Nepusz and Petróczi (2013).

${ }^{3}$ Note that the recent game theoretic literature on doping has utilized contest theory to analyze settings where, amongst other things, effort is also a choice variable (e.g. Krakel 2007; Ryvkin 2013; Mohan and Hazari 2016). Here we focus on the performance enhancing drug game to make the analysis as transparent as possible.
} 
Berentsen (2002) shows that if we have two athletes who differ a lot in ability then there will exist a no doping equilibrium independent of any social norms (see also Breivik 1992). Even so, it is important to question whether the results to follow are sensitive to the assumption of symmetry and so I will comment on this as we proceed.

\section{Inequality aversion}

In this section I work briefly through the model of inequality aversion due to Fehr and Schmidt (1999). Let me highlight that this model does not take account of beliefs and so is included here primarily as a comparator for the psychological game theory models that follow in Sections 5 and 6. The Fehr and Schmidt (1999) model provides a useful benchmark for two reasons. Most importantly, the approach used by Eber (2008) is closely related to that of inequality aversion and so working through the Fehr and Schmidt (1999) model allows me to summarize the main findings of Eber (2008). Also, there are many comparisons in the literature between inequality aversion and theories based on psychological game theory (e.g. Bacharach, Guerra and Zizzo 2007; Falk, Fehr and Fischbacher 2008; Xiao and Bicchieri 2010) and so it is interesting to also provide that comparison here.

The starting point for inequality aversion is that the 'fairest' outcome is one in which both players have the same expected payoff. Given that both athletes are symmetric this seems reasonable. An athlete then experiences disutility if they earn less than others (which could reflect envy) or more than others (which could reflect guilt). In interpreting aversion from earning more than others it is important to recognize that inequality aversion is based on payoffs and not competitive outcomes. So, it is not so much athletes do not want to win but that they may not want excessive monetary reward for winning. ${ }^{4}$ Parameters $\alpha_{i}$ and $\beta_{i}$ measure the sensitivity of athlete $i$ to earning less and more than others, where $\alpha_{i} \geq \beta_{i} \geq 0$. Taking inequality aversion into account transforms the standard performance-enhancing drug game to the one depicted in Table 2. If both athletes dope or both do not dope then they get the same material payoff and so there is no inequality. If, by contrast, athlete 1 dopes and athlete 2 does not then athlete 1 has a material payoff advantage of $p w-h-(1-p) w=w(2 p-1)-h$. Denote this difference $D$. Athlete 1 's payoff then consists of his material payoff, $p w-h$, and disutility from having gained an advantage, $\beta_{1} D$. Athlete 2's payoff consists of his material payoff, $(1-p) w$, and disutility from being at a disadvantage, $\alpha_{2} D$. A similar logic treats the case where athlete 2 dopes and athlete 1 does not.

\section{Athlete 2}

$\mathrm{D}$

$\mathrm{ND}$

$\begin{array}{cccc}\text { Athlete } 1 & \mathrm{D} & w / 2-h, w / 2-h & p w-h-\beta_{1} D,(1-p) w-\alpha_{2} D \\ & \mathrm{ND} & (1-p) w-\alpha_{1} D, p w-h-\beta_{2} D & w / 2, w / 2\end{array}$

Table 2: The performance-enhancing drug game with inequality aversion

\footnotetext{
${ }^{4}$ Inequality aversion is less well suited to asymmetric situations in which one athlete might naturally be expected to earn a higher payoff than his competitor.
} 
The key insight from Eber (2008) is that inequality aversion can transform the performance-enhancing drug game into a coordination game. In particular, action profile $(0,0)$ will be a Nash equilibrium if

$$
\frac{w}{2} \geq p w-h-\beta_{i}(w(2 p-1)-h)
$$

for $i=1,2$. This reduces to condition

$$
\beta_{1}, \beta_{2} \geq \frac{\left(p-\frac{1}{2}\right) w-h}{2\left(p-\frac{1}{2}\right) w-h} .
$$

Note that action profile $(1,1)$ remains a Nash equilibrium. Inequality aversion, therefore, can generate a coordination game in which it is an equilibrium for neither to dope and an equilibrium for both to dope.

In order to gain further insight let us look in more detail at the incentives of athlete 1 . Suppose that athlete 2 dopes with probability $q$. Then the expected payoff of athlete 1 if he dopes is $q w / 2+(1-q)\left(p w-\beta_{1} D\right)-h$. His expected payoff if he does not dope is $q\left((1-p) w-\alpha_{1} D\right)+(1-q) w / 2$. Hence, it is optimal for athlete 1 to dope if

$$
\frac{\beta_{1}}{\alpha_{1}+\beta_{1}}-\frac{w\left(p-\frac{1}{2}\right)-h}{D\left(\alpha_{1}+\beta_{1}\right)}=\bar{q}<q .
$$

Note that $\bar{q}<1 / 2$, because $w(p-1 / 2)>h$ and $\alpha_{1} \geq \beta_{1}$. It is, therefore, optimal for athlete 1 to dope if he thinks athlete 2 will dope with probability $1 / 2$ or more. This means that the doping equilibrium $(1,1)$ is risk dominant (Eber 2008). Equilibrium $(0,0)$ is payoff dominant. If, therefore, condition (1) is satisfied we have a stag-hunt coordination game. ${ }^{5}$

Before we move on let me relate the above analysis to that of Eber (2008). Rather than look at inequality in payoffs (as in the Fehr and Scmhidt (1999) model) Eber (2008) focuses on inequality in the probability of winning. In this case an athlete feels averse to gaining an 'unfair' competitive advantage or seeing the other athlete gain an unfair advantage. This provides a different interpretation of fairness to that of Fehr and Schmidt (1999). But in practical terms it is equivalent to setting $D=(p-1 / 2) w$ in Table 2, with an appropriate reinterpretation of $\alpha_{i}$ and $\beta_{i}$. All the analysis discussed above, thus, follows through in an almost identical fashion to that of Eber (2008), except condition (1) is

$$
\beta_{1}, \beta_{2} \geq \frac{\left(p-\frac{1}{2}\right) w-h}{\left(p-\frac{1}{2}\right) w} .
$$

Not surprisingly, the findings of Eber (2008) are, thus, unchanged whether we consider inequality aversion in payoffs or fairness based on unfair advantage. ${ }^{6}$

\footnotetext{
${ }^{5}$ The evidence on behavior in games with Pareto ranked equilibria is mixed in terms of which equilibrium is most likely to emerge (Devetag and Ortmann 2007). It is important to recognize, however, that in terms of material payoffs the performance-enhancing drug game is still a prisoners dilemma and so the experimental evidence on linear public good games is also relevant (e.g. Wolff 2017). Again, the evidence is mixed (Chaudhuri 2011). One can, however, note that inequality aversion is often given as an explanation for cooperation in public good games (e.g. Fehr and Schmidt 1999).

${ }^{6}$ More generally, one can envisage settings where doping may dramatically increase an athletes chance of winning but make little difference to payoff inequality and vice-versa. Hence the difference may matter. The main reason it does not do so here is the assumption of symmetry.
} 
The preceding analysis shows that a norm, whether motivated by inequality or otherwise, can result in a no doping equilibrium. Arguably, however, the story is not that compelling. First, we require both athletes to dislike having a payoff advantage. Experimental evidence does suggest that many people dislike advantageous inequality (e.g. Bellemare, Kröger and Van Soest 2008; Beranek, Cubitt and Gächter 2015, Yang, Onderstal and Schram 2016). Even so, it only takes one athlete to be self focused for the no doping equilibrium to unravel. And the 'win at all costs' approach of athletes (Krumer, Shavit and Rosenboim 2011) suggests this is likely. Second, we require the athletes to 'coordinate' in the sense that they converge to the no doping equilibrium. The preceding analysis, for instance, gives limited insight on what will happen if the two athletes have wrong beliefs about the actions of the other. Third, inequality aversion is highly dependent on symmetry. If the athletes start unequal, because one has a higher ability, then the fairest outcome may not be one in which both have the same material payoff.

Given the various issues mentioned in the preceding discussion it seems vital to explore, in more depth, the extent to which social norms can realistically incentivize an athlete to not dope. This motivates the analysis to follow in which we consider alternative models of social preferences. While inequality aversion provides a simple, reduced form model, it has been argued that psychological game theory offers a more nuanced approach (Camerer 2003; Sobel 2005; Battigali and Dufwenberg 2009; Cartwright 2014). In the following I will look at models of reciprocity and guilt aversion to see if any extra insight can be gained on the ways in which doping can be reduced.

\section{Reciprocity}

In this section I will work through the model of fairness due to Rabin (1993) (drawing also on Dufwenberg and Kirchsteiger 2004). The key underlying idea is that an athlete will want to reciprocate the kindness (or unkindness) of the other athlete. If, for instance, athlete 2 does not dope then this is kind because it increases the expected payoff of athlete 1 . Athlete 1 may desire to reciprocate this act of kindness by also not doping. On the flip side, if athlete 2 dopes then this is unkind and athlete 2 may desire to reciprocate by also doping. Note that this notion of reciprocation is broadly consistent with the notion of fairness used by Eber (2008), whereby an athlete considers it 'fair' to use drugs if her competitor also does but 'unfair' to do so if her competitor does not take drugs. It is also consistent with the evidence discussed earlier that athletes willingness to use drugs appears to be positively correlated with first-order beliefs on the doping of others.

To introduce the model more formally let us work through a specific scenario that leads to a no doping equilibrium. Suppose that athlete 1 believes that athlete 2 will not dope, i.e. $b_{12}=0$. Given this belief, athlete 1 expects that by choosing to dope the monetary payoff of athlete 2 will be $(1-p) w$. Also, he expects that by choosing not to dope the monetary payoff of athlete 2 will be $w / 2$. Following Rabin (1993: p. 1286) the equitable payoff of athlete 2 is then calculated as the midpoint 
between these extremes ${ }^{7}$

$$
\pi_{2}^{e}\left(b_{12}=0\right)=\frac{(1-p) w+\frac{w}{2}}{2}=\frac{(3-2 p) w}{4} .
$$

The kindness of athlete 1 towards athlete 2 if he does not dope is then given by the difference between athlete 2's received and equitable payoff

$$
k_{12}\left(a_{1}=0, b_{12}=0\right)=\frac{w}{2}-\frac{(3-2 p) w}{4}=\frac{\left(p-\frac{1}{2}\right) w}{2} .
$$

That this value is positive shows that athlete 1 is being kind towards athlete 2 by not doping. The kindness of athlete 1 towards athlete 2 if he does dope is given by

$$
k_{12}\left(a_{1}=1, b_{12}=0\right)=(1-p) w-\frac{(3-2 p) w}{4}=-\frac{\left(p-\frac{1}{2}\right) w}{2} .
$$

That this value is negative shows that athlete 1 is being unkind towards athlete 2 by doping.

We next consider athlete 1's belief about how kind athlete 2 is being towards him. This will depend on athlete 1's second-order beliefs. Suppose that $c_{121}=0$ and so athlete 1 believes athlete 2 expects him to not dope. Then, retaining the assumption that $b_{12}=0$, athlete 1 's belief about the kindness of athlete 2 is given by

$$
\lambda_{121}\left(b_{12}=0, c_{121}=0\right)=k_{21}\left(b_{12}=0, c_{121}=0\right)=\frac{\left(p-\frac{1}{2}\right) w}{2} .
$$

This value is positive because athlete 1 believes that athlete 2 will not dope and is, therefore, being kind.

The overall expected payoff of athlete 1 if he does not dope is then given by

$$
\pi_{1}\left(a_{1}=0, b_{12}=0, c_{121}=0\right)=\frac{w}{2}+r_{1} k_{12} \lambda_{121}=\frac{w}{2}+r_{1} \frac{\left(p-\frac{1}{2}\right)^{2} w^{2}}{4}
$$

where $r_{1}$ measures athlete 1 's sensitivity to reciprocity. The basic idea here is that athlete 1 wants to be kind because he believes that athlete 2 is being kind. His expected payoff if he dopes is given by

$$
\pi_{1}\left(a_{1}=1, b_{12}=0, c_{121}=0\right)=p w-h+r_{1} k_{12} \lambda_{121}=p w-h-r_{1} \frac{\left(p-\frac{1}{2}\right)^{2} w^{2}}{4} .
$$

In this case athlete 1 experiences disutility from failing to reciprocate athlete 2's kindness.

We can see that athlete 1 gets a higher payoff from not doping (given his beliefs) if and only if ${ }^{8}$

$$
r_{1}>\frac{2}{\left(p-\frac{1}{2}\right) w}\left(1-\frac{h}{\left(p-\frac{1}{2}\right) w}\right)>0 .
$$

A fairness equilibrium (as defined by Rabin 1993) exists when each athlete maximizes his payoff given his beliefs and those beliefs are correct. ${ }^{9}$ So, a no doping equilibrium

\footnotetext{
${ }^{7}$ One could consider alternatives to this definition of equitable payoff. For instance, Falk and Fischbacher (2006) associate equitable payoff with everyone getting the same payoff.

${ }^{8}$ Recall that $(p-1 / 2) w>h$ and so we require $r_{1}>0$.

${ }^{9}$ Fairness equilibrium is the natural extension of Nash equilibrium to psychological games.
} 
exists (where $a_{1}=b_{12}=c_{121}=0$ and $a_{2}=b_{21}=c_{212}=0$ ) if both athletes are sufficiently sensitive to reciprocity. Note that a doping equilibrium would also still exist (where $a_{1}=b_{12}=c_{121}=1$ and $a_{2}=b_{21}=c_{212}=1$ ) for any values of $r_{1}$ and $r_{2}$. Moreover, it is simple to check that there cannot exist an equilibrium where one athlete dopes and the other does not. ${ }^{10}$ The reciprocity model, therefore, gives very similar results to that of inequality aversion in terms of equilibrium predictions.

In order to consider non-equilibrium predictions, let us generalize the preceding discussion and simply say that athlete 1 believes that athlete 2 will dope with probability $b_{12}$. Given this belief, athlete 1 expects that by choosing to dope the monetary payoff of athlete 2 will be $\left(1-b_{12}\right)(1-p) w+b_{12} w / 2$. Also, he expects that by choosing not to dope the monetary payoff will be $\left(1-b_{12}\right) w / 2+b_{12} p w$. The equitable payoff is, therefore,

$\pi_{2}^{e}\left(b_{12}\right)=\frac{\left(1-b_{12}\right)(1-p) w+b_{12} \frac{w}{2}+\left(1-b_{12}\right) \frac{w}{2}+b_{12} p w}{2}=\frac{(3-2 p) w}{4}+b_{12} w\left(p-\frac{1}{2}\right)$.

The kindness of athlete 1 towards athlete 2 if he does not dope is then given by the difference between athlete 2's received payoff and the equitable payoff

$$
k_{12}\left(a_{1}=0, b_{12}\right)=\left(1-b_{12}\right) \frac{w}{2}+b_{12} p w-\frac{(3-2 p) w}{4}-b_{12} w\left(p-\frac{1}{2}\right)=\frac{\left(p-\frac{1}{2}\right) w}{2} .
$$

The kindness of athlete 1 towards athlete 2 if he dopes is given by $k_{12}\left(a_{1}=1, b_{12}\right)=$ $-(p-1 / 2) w / 2$. Crucially, note that kindness does not depend on first-order beliefs. Similarly, athlete 1's belief about the kindness of athlete 2 will not depend on secondorder beliefs.

The overall expected payoff of athlete 1 if he does not dope is, therefore, given by

$$
\begin{aligned}
\pi_{1}\left(a_{1}=0, b_{12}, c_{121}\right) & =b_{12}\left((1-p) w-r_{1} \frac{\left(p-\frac{1}{2}\right)^{2} w^{2}}{4}\right)+\left(1-b_{12}\right)\left(\frac{w}{2}+r_{1} \frac{\left(p-\frac{1}{2}\right)^{2} w^{2}}{4}\right) \\
& =\frac{w}{2}-b_{12} w\left(p-\frac{1}{2}\right)+r_{1}\left(1-2 b_{12}\right) \frac{\left(p-\frac{1}{2}\right)^{2} w^{2}}{4} .
\end{aligned}
$$

Note that his expected payoff does depend on first-order belief $b_{12}$. His expected payoff if he dopes is given by

$$
\pi_{1}\left(a_{1}=1, b_{12}, c_{121}\right)=\frac{w}{2}+\left(1-b_{12}\right) w\left(p-\frac{1}{2}\right)+r_{1}\left(2 b_{12}-1\right) \frac{\left(p-\frac{1}{2}\right)^{2} w^{2}}{4}-h .
$$

It is, therefore, optimal for athlete 1 to dope (assuming $r_{1}>0$ ) if

$$
\frac{1}{2}-\frac{w\left(p-\frac{1}{2}\right)-h}{r_{1}\left(p-\frac{1}{2}\right)^{2} w^{2}}=\bar{b}<b_{12}
$$

Given that $\bar{b}<1 / 2$ it is optimal for athlete 1 to dope if he believes that athlete 2 will dope with probability $1 / 2$ or more.

\footnotetext{
${ }^{10}$ Consider, for instance, the situation where athlete 1 dopes and athlete 2 does not. Athlete 1 is being unkind while athlete 2 is being kind. It follows that athlete 2 would have an incentive to dope.
} 
This latter finding is similar to the result obtained with inequality aversion. Here, though, we gain additional insight because beliefs are explicitly modeled. The interesting thing to observe is that second-order beliefs drop out of the picture. In interpretation this means that athlete 1 is indifferent to the beliefs of athlete 2 . He does not, for instance, consider it less unkind that athlete 2 would dope because he expects others to dope. Doping is unkind, no matter what the cause. This means that an athlete's incentives to dope are driven solely by first-order beliefs on the likely doping intention of the other athlete. The more pessimistic his belief, however correct that belief may be, the more incentive to dope. This raises the fundamental question of factors that influence beliefs. We discuss this more in Section 7 but mention here that a range of factors including feedback from coaches, teammates and the media are likely to matter. And, as pointed out by a reviewer, past experience may be especially pivotal. In particular, an athlete who has lost previous competitions may form pessimistic first-order beliefs. This would suggest that less able athletes may be more prone to doping (because they form pessimistic first-order beliefs).

So what have we learned from the reciprocity based model of fairness? The main lesson appears to be that the results from Eber (2008) are extendable to a model that (a) takes full account of beliefs and (b) is based on reciprocity rather than inequality aversion. This speaks to the robustness of the findings of Eber (2008). Furthermore, the analysis of this section is not dependent on symmetry because kindness is judged relative to an equitable payoff that is based on the maximum and minimum material payoff an athlete could earn. The analysis of this section, thus, readily extends to settings with asymmetric ability. Difficult questions remain, however, about how readily doping can be reduced. In particular, a no doping equilibrium requires both athletes to value reciprocity. Moreover, we see the critical role of first-order beliefs as captured in equation (4) - an athlete is only going to refrain from doping if he is optimistic that the other athlete will also refrain from doping. So, we need both athletes to be 'optimistic reciprocators'

\section{Guilt Aversion}

In this section we turn to a model of guilt aversion based on Charness and Dufwenberg (2006) (see also Battigalli and Dufwenberg 2007, 2009; Cartwright 2018). Guilt aversion pays particular attention to second-order beliefs and the idea of 'letting someone down'. If an athlete does not dope then this may be unexpected but is clearly not a reason for guilt. If, however, an athlete dopes then he may experience guilt. The size of guilt is assumed to depend crucially on the athlete's beliefs about what he was expected to do. For instance, if $c_{121}=1$ then athlete 1 believes he was expected to dope and so has no reason to feel guilt if he does dope. But, if $c_{121}=0$ then athlete 1 believes he was not expected to dope and so will experience guilt.

The standard framework of Battigalli and Dufwenberg (2007) assumes that the extent of guilt athlete 1 experiences will depend on the difference between the realized and expected payoff of athlete 2. So, the athlete feels guilt at letting his competitor down. This is an assumption that would seem at odds with the evidence athletes express greater guilt at letting down friends, family and teammates, rather than competitors (e.g. Bloodworth and McNamee 2010; Overbye et al. 2013). Note, however, that the zero-sum nature of the prize allocation means that the amount 
athlete 2 looses is equal to the amount athlete 1 gains. So, we can equivalently say that athlete 1 feels guilt in proportion to the amount he gains by doping or the amount athlete 2 loses. Moreover, in interpretation, we can think of $c_{121}$ as measuring athlete 1's overall belief about the expectations of others, including friends and team mates. ${ }^{11}$

To formally explore the consequences of guilt aversion let us begin by looking for a no doping equilibrium. So, consider action profile $(0,0)$ and second-order beliefs $c_{121}=c_{212}=0$. Expected payoffs are $w / 2$ for both athletes. If athlete 1 were to 'deviate from what was expected' and dope then athlete 2's payoff would drop to $(1-p) w$. The overall drop in payoff is $w / 2-(1-p) w=w(p-1 / 2)$. The expected payoff of athlete 1 is then given by

$$
g_{1}\left(a_{1}=1, b_{12}=0, c_{121}=0\right)=p w-h-\gamma_{1} w\left(p-\frac{1}{2}\right)
$$

where $\gamma_{1}$ is athlete 1's sensitivity to guilt. So, athlete 1 maximizes his payoff by not doping if and only if

$$
1-\frac{h}{w\left(p-\frac{1}{2}\right)}<\gamma_{1}
$$

If, therefore, guilt aversion is sufficiently strong athlete 1 does not have an incentive to dope. Which means that if both athletes are sufficiently guilt averse there exists a no doping equilibrium.

The existence of a no doping equilibrium is clearly consistent with the results derived with inequality aversion and reciprocity. From here on, however, things begin to diverge. To see why, suppose that athlete 1 believes athlete 2 will dope with probability $b_{12}$. Then athlete 2 's expected payoff if athlete 1 does not dope is $\left(1-b_{12}\right) w / 2+q(p w-h)$ and athlete 2's expected payoff if athlete 1 dopes is $\left(1-b_{12}\right)(1-p) w+q(w / 2-h)$. Note that the difference is again $w(p-1 / 2)$. The guilt that athlete 1 experiences will depend on this payoff difference and his secondorder beliefs $c_{121}$. In particular, the expected payoff of athlete 1 if he dopes is equal to

$$
g_{1}\left(a_{1}=1, b_{12}, c_{121}\right)=\left(1-b_{12}\right) p w+\frac{b_{12} w}{2}-h-\gamma_{1}\left(1-c_{121}\right) w\left(p-\frac{1}{2}\right) .
$$

If $c_{121}=1$ and so athlete believes he was expected to dope then he experiences no guilt. If $c_{121}=0$ then he experiences maximum guilt. Guilt, therefore, critically depends on second-order beliefs. Herein we see why guilt cannot be modeled merely as a fixed cost from doping. ${ }^{12}$

If he does not dope the expected payoff of athlete 1 is $\left(1-b_{12}\right) w / 2+b_{12}(1-p) w$. Comparing respective payoffs we can see that it is optimal for athlete 1 to dope if and only if

$$
\frac{1}{\gamma_{1}}-\frac{h}{\gamma_{1} w\left(p-\frac{1}{2}\right)}-1<c_{121} .
$$

\footnotetext{
${ }^{11}$ In a model of reciprocity this interpretation would not be valid because the kindness of athlete 2 is, in principle, based on what he believed athlete 1 would do.

${ }^{12}$ Even if we move away from a belief based model of guilt aversion it is still insufficient to model guilt as a fixed cost. For instance, guilt may be influenced by norms which then depend on a reference point. Such a model would give similar results to that of the belief based model (Cartwright 2018).
} 
Note that if $c_{121}=0$ then this condition reduces to condition (5). It is, therefore, optimal for athlete 1 to not dope if he believes he is expected to not dope and his sensitivity to guilt aversion is sufficiently strong. Crucially, athlete 1's incentive to dope is independent of his first-order belief. In particular, it may be optimal for athlete 1 to not dope even if he believes athlete 2 will dope. This outcome is consistent with the evidence that guilt is a strong predictor of doping intentions, independent of the actions of others (e.g. Bloodworth and McNamee 2010; Barkoukis et al. 2014).

The preceding analysis suggests that guilt aversion can result in a relatively strong incentive to not dope. We do not, as is the case with inequality aversion or reciprocity, need athletes to coordinate on a risk dominated equilibrium. We simply need an athlete to believe others expect him to not dope and to feel guilt from disappointing this expectation. Also, the analysis does not depend on symmetry because guilt is measured by changes in absolute payoff. Note, however, that a doping equilibrium, in which both athletes are expected to dope and so feel no guilt from doping, still exists. It is also possible to have an equilibrium in which, say, athlete 1 does not dope, because he would experience guilt from doing so, while athlete 2 does dope. Guilt aversion does not, therefore, necessarily result in no doping, even if the athletes are strongly guilt averse. Again, beliefs will matter. Doping incentives, however, become more focused on the individual and what he believes others expect of him than on the broader competition between athletes. This could be crucial, as we shall now discuss, when we look at ways to combat doping.

\section{Conclusion}

This paper explores the extent to which social norms can overcome incentives to dope in sport. Three different models of social norms are considered and compared. These are a model of inequality aversion, closely related to that considered by Eber (2008), a model of reciprocity (Rabin 1993; Dufwenberg and Kirchsteiger 2004) and finally a model of guilt aversion (Battigalli and Dufwenberg 2007). The latter two models fit within the framework of psychological game theory and so allow us to take explicit account of beliefs. We see that the models of inequality aversion and reciprocity give very similar results and suggest that incentives to dope will depend on first-order beliefs regarding the intentions of others to dope. The model of guilt aversion gives contrasting results and suggests incentives to dope will depend on second-order beliefs regarding whether an athlete believes others expect him to dope.

All three models show that norms can support an equilibrium where athletes refrain from doping. Beliefs, however, will prove crucial. In particular, reciprocity can only work if athletes believe competitors will race clean while guilt aversion can only work if athletes believe others expect them to race clean. In reality we can, as discussed in Section 2, expect both reciprocity and guilt aversion to be important determinants of behavior and so first and second-order beliefs will likely matter. Moreover, it is beliefs rather than reality which will drive behavior. This all suggests that it is essential that the fight against doping should pay attention to clean athletes rather than become (or continue to be) a narrative dominated by doping scandal (Englar-Carlson et al. 2016). 
Inequality aversion and reciprocity convert the performance-enhancing drug game into a coordination game. While this does lead to the existence of an equilibrium with no doping it is far from clear how easily this equilibrium can be reached. It only needs, for instance, one athlete to dope, or to believe others will dope, for things to unravel. Indeed, inequality aversion or reciprocity means that if one athlete dopes others have even more incentive to dope because they dislike the 'unfair' advantage obtained by the doper (Eber 2011). If, therefore, inequality aversion or reciprocity are to work a substantial transformation in beliefs is required. Arguably this has happened in cycling where a sport rife with doping has become clean (LentillonKaestner, Hagger and Hardcastle 2012). It is questionable, though, how easily this example could be replicated in other domains.

Guilt aversion works differently in that an athlete may desire to race clean irrespective of what competitors do. This is because the athlete feels guilt from letting down the expectations of others. Crucially, survey evidence suggests that letting down others is a strong motivation against doping (e.g. Strelan and Boeckmann 2006; Barkoukis et al. 2014). It may, therefore, be possible to reduce doping without a transformation of overall beliefs. Instead, individual athletes or teams of athletes can be targeted. This is particularly relevant for national bodies or coaches who have little control over the tendency of athletes outside their remit to dope but can influence the second-order beliefs of their own athletes. The success of this more individual approach will still, however, rely on the support of governing bodies, particularly if clean athletes are likely to be less successful than dopers.

The absence of universal data on the prevalence of doping in sport means that athletes will form their own subjective beliefs. Given the bias towards reporting doping scandals it would not be a surprise if athletes form overly pessimistic beliefs about doping in sport. And note that this can generate a self-fulfilling tendency to dope (see, for instance, Bacharach et al. 2007). It would appear critical, therefore, that athletes are offered positive, as well as negative, information regarding doping and the expectations for clean sport. Clearly, it would be naive to think that a positive vision will eliminate doping in sport. A more positive vision may, however, have a pronounced effect on individual incentives and in so doing reduce the prevalence of doping (Schultz et al. 2007). The analysis of this paper shows that it is particularly important to instill in athletes a (second-order) belief that they are not expected to dope. There are practical measures that coaches, family and governing bodies could do to bring this about, as seemingly evidenced by a transformation in attitudes in road cycling.

This is not to say that the authorities should not aggressively try to eliminate doping through testing. A balance, however, is needed. For instance, the current whereabouts system of out-of-competition sampling together with strict liability rules on doping give an impression of 'guilty until proven innocent'. This 'presumption of guilt' may influence beliefs, particularly, second-order beliefs in an undesirable way. It is important, therefore, to recognize the role played by social norms. To sum this up let me end with some quotes that I would endorse. Bird and Wagner (1997, p. 755) write 'the only real hope for ending the practice of doping lies in the norms of fair competition among the athletes. Yet there is hope.' This hope, though, requires a blending of formal and informal sanctions because '[n]othing damages informal norms more than the presence of a failed system of formal laws.' Or to look from a different angle, Strelan and Boeckmann (2006, p. 2925) write, '[i]t appears 
that legal sanctions are salient only to the extent that they convey information about the moral and health costs associated with using banned drugs.'

\section{References}

[1] Anshel, M. H. (1991). A survey of elite athletes on the perceived causes of using banned drugs in sport. Journal of Sport Behavior, 14(4), 283.

[2] Bacharach, M., Guerra, G., \& Zizzo, D. J. (2007). The self-fulfilling property of trust: An experimental study. Theory and Decision, 63(4), 349-388.

[3] Barkoukis, V., Lazuras, L., \& Tsorbatzoudis, H. (2014). Beliefs about the causes of success in sports and susceptibility for doping use in adolescent athletes. Journal of Sports Sciences, 32(3), 212-219.

[4] Beranek, B., Cubitt, R., \& Gächter, S. (2015). Stated and revealed inequality aversion in three subject pools. Journal of the Economic Science Association, $1(1), 43-58$.

[5] Battigalli, P., \& Dufwenberg, M. (2009). Dynamic psychological games. Journal of Economic Theory, 144(1), 1-35.

[6] Battigalli, P., \& Dufwenberg, M. (2007). Guilt in games. American Economic Review, 97(2), 170-176.

[7] Bellemare, C., Kröger, S., \& Van Soest, A. (2008). Measuring inequity aversion in a heterogeneous population using experimental decisions and subjective probabilities. Econometrica, 76(4), 815-839.

[8] Berentsen, A. (2002). The economics of doping. European Journal of Political Economy, 18(1), 109-127.

[9] Bergstrom, R. L., \& Neighbors, C. (2006). Body image disturbance and the social norms approach: An integrative review of the literature. Journal of Social and Clinical Psychology, 25(9), 975-1000.

[10] Bicchieri, C. (2018). Norms in the wild: How to diagnose, measure, and change social norms. Oxford University Press.

[11] Bird, E. J., \& Wagner, G. G. (1997). Sport as a Common Property Resource A Solution to the Dilemmas of Doping. Journal of Conflict Resolution, 41(6), 749-766.

[12] Bloodworth, A., \& McNamee, M. (2010). Clean Olympians? Doping and antidoping: The views of talented young British athletes. International Journal of Drug Policy, 21(4), 276-282.

[13] Breivik, G. (1992). Doping games a game theoretical exploration of doping. International Review for the Sociology of Sport, 27(3), 235-253.

[14] Camerer, C. (2003). Behavioral game theory: Experiments in strategic interaction. Princeton University Press. 
[15] Cartwright, E. (2018). A survey of belief-based guilt aversion in trust and dictator games' Journal of Economic Behavior and Organization, forthcoming.

[16] Cartwright, E. (2014). Behavioral economics (2nd Edition). Routledge.

[17] Chaudhuri, A. (2011). Sustaining cooperation in laboratory public goods experiments: a selective survey of the literature. Experimental Economics, 14(1), $47-83$.

[18] Charness, G., \& Dufwenberg, M. (2006). Promises and partnership. Econometrica, 74(6), 1579-1601.

[19] Devetag, G., \& Ortmann, A. (2007). When and why? A critical survey on coordination failure in the laboratory. Experimental Economics, 10(3), 331-344.

[20] Dilger, A., Frick, B., \& Tolsdorf, F. (2007). Are athletes doped? Some theoretical arguments and empirical evidence. Contemporary Economic Policy, 25(4), 604-615.

[21] Dufwenberg, M., \& Kirchsteiger, G. (2004). A theory of sequential reciprocity. Games and Economic Behavior, 47(2), 268-298.

[22] Dufwenberg, M., Gächter, S., \& Hennig-Schmidt, H. (2011). The framing of games and the psychology of play. Games and Economic Behavior, 73(2), 459478.

[23] Eber, N. (2011). Fair play in contests. Journal of Economics, 103(3), 253-270.

[24] Eber, N. (2008). The Performance-Enhancing Drug Game Reconsidered. Journal of Sports Economics, 9(3), 318-327.

[25] Englar-Carlson, M., Gleaves, J., Macedo, E., \& Lee, H. (2016). What about the clean athletes? The need for positive psychology in anti-doping research. Performance Enhancement \& Health, 4(3), 116-122.

[26] Falk, A., Fehr, E., \& Fischbacher, U. (2008). Testing theories of fairnessIntentions matter. Games and Economic Behavior, 62(1), 287-303.

[27] Falk, A., \& Fischbacher, U. (2006). A theory of reciprocity. Games and Economic Behavior, 54(2), 293-315.

[28] Fehr, E., \& Schmidt, K. M. (1999). A theory of fairness, competition, and cooperation. The Quarterly Journal of Economics, 114(3), 817-868.

[29] Geanakoplos, J., Pearce, D., \& Stacchetti, E. (1989). Psychological games and sequential rationality. Games and economic Behavior, 1(1), 60-79.

[30] Goulet, C., Valois, P., Buist, A., \& Côté, M. (2010). Predictors of the use of performance-enhancing substances by young athletes. Clinical Journal of Sport Medicine, 20(4), 243-248.

[31] Haugen, K. K. (2004). The performance-enhancing drug game. Journal of Sports Economics, 5(1), 67-86. 
[32] Haugen, K. K., Nepusz, T., \& Petróczi, A. (2013). The multi-player performance-enhancing drug game. PloS one, 8(5), e63306.

[33] Huybers, T., \& Mazanov, J. (2012). What would Kim do: a choice study of projected athlete doping considerations. Journal of Sport Management, 26(4), 322-334.

[34] Jalleh, G., Donovan, R. J., \& Jobling, I. (2014). Predicting attitude towards performance enhancing substance use: a comprehensive test of the Sport Drug Control Model with elite Australian athletes. Journal of Science and Medicine in Sport, 17(6), 574-579.

[35] Kavussanu, M. (2008). Moral behaviour in sport: A critical review of the literature. International Review of Sport and Exercise Psychology, 1(2), 124-138.

[36] Kirby, K., Moran, A., \& Guerin, S. (2011). A qualitative analysis of the experiences of elite athletes who have admitted to doping for performance enhancement. International Journal of Sport Policy and Politics, 3(2), 205-224.

[37] Kräkel, M. (2007). Doping and cheating in contest-like situations. European Journal of Political Economy, 23(4), 988-1006.

[38] Lentillon-Kaestner, V., Hagger, M. S., \& Hardcastle, S. (2012). Health and doping in elite-level cycling. Scandinavian Journal of Medicine 8 Science in Sports, 22(5), 596-606.

[39] McClaren, R. H. (2016). Independent person WADA investigation of Sochi allegation. WADA Report.

[40] Mohan, V., \& Hazari, B. (2016). Cheating in contests: Anti-doping regulatory problems in sport. Journal of Sports Economics, 17(7), 736-747.

[41] Morente-Sánchez, J., \& Zabala, M. (2013). Doping in sport: a review of elite athletes' attitudes, beliefs, and knowledge. Sports Medicine, 43(6), 395-411.

[42] Ntoumanis, N., Ng, J. Y., Barkoukis, V., \& Backhouse, S. (2014). Personal and psychosocial predictors of doping use in physical activity settings: a metaanalysis. Sports Medicine, 44(11), 1603-1624.

[43] Overbye, M., Knudsen, M. L., \& Pfister, G. (2013). To dope or not to dope: Elite athletes' perceptions of doping deterrents and incentives. Performance Enhancement and Health, 2(3), 119-134.

[44] Perkins, H. W. (2002). Social norms and the prevention of alcohol misuse in collegiate contexts. Journal of Studies on Alcohol, (14), 164-172.

[45] Petróczi, A., Naughton, D. P., Mazanov, J., Backhouse, S. H., \& Nepusz, T. (2008). Comfort in big numbers: Does over-estimation of doping prevalence in others indicate self-involvement?. Journal of Occupational Medicine and Toxicology, 3(1), 19.

[46] Rabin, M. (1993). Incorporating fairness into game theory and economics. American economic review, 1281-1302. 
[47] Ryvkin, D. (2013). Contests with doping. Journal of Sports Economics, 14(3), 253-275.

[48] Schultz, P. W., Nolan, J. M., Cialdini, R. B., Goldstein, N. J., \& Griskevicius, V. (2007). The constructive, destructive, and reconstructive power of social norms. Psychological Science, 18(5), 429-434.

[49] Sobel, J. (2005). Interdependent preferences and reciprocity. Journal of Economic Literature, 43(2), 392-436.

[50] Strelan, P., \& Boeckmann, R. J. (2006). Why Drug Testing in Elite Sport Does Not Work: Perceptual Deterrence Theory and the Role of Personal Moral Beliefs. Journal of Applied Social Psychology, 36(12), 2909-2934.

[51] Wolff, I. (2017). What are the equilibria in public-good experiments?. Economics Letters, 150, 83-85.

[52] Xiao, E., \& Bicchieri, C. (2010). When equality trumps reciprocity. Journal of Economic Psychology, 31(3), 456-470.

[53] Yang, Y., Onderstal, S., \& Schram, A. (2016). Inequity aversion revisited. Journal of Economic Psychology, 54, 1-16. 